\title{
Evaluation of 3 Human Cervical Fusion Implants for use in the Canine Cervical Vertebral Column
}

\begin{tabular}{|r|l|}
\hline Journal: & Veterinary Surgery \\
\hline Manuscript ID & VSU-15-229.R1 \\
\hline Manuscript Type: & Original Article - Research \\
\hline Keywords: & dog, cervical vertebral column, interbody cage, Zero-P, Uniplate \\
\hline \multicolumn{2}{|c}{} \\
\hline
\end{tabular}

\section{SCHOLARONE}

Manuscripts 


\section{ABSTRACT}

2 Objective: To assess technical feasibility and mechanical properties of three locking

3 plate designs (Zero-P, Zero-P VA and Uniplate 2) for use in the canine cervical spine.

4 Study Design: Prospective ex-vivo study using canine cadaveric tissues.

5 Animals: Eighteen canine cervical spines collected from skeletally mature

6 large-breed dogs.

7 Methods: Specimens were screened using radiography and allocated into balanced

8 groups based on bone density. Stiffness of intact C4-C5 vertebral motion units (VMU)

9 was measured in extension, flexion and lateral bending using non-destructive 4-point

10 bend testing. Uniplate 2 was then implanted at $\mathrm{C} 4-\mathrm{C} 5$ and mechanical testing was

11 repeated. Mechanical test data were compared against those from six spines implanted

12 with monocortical screws, an allograft ring spacer and PMMA.

13 Results: The Zero-P and Zero-P VA systems could not be surgically implanted due to

14 anatomical constraints of the canine cervical spine. Fixation with Uniplate 2 or with

15 screws/PMMA significantly increased stiffness of the C4-C5 VMU compared to

16 unaltered specimens $(\mathrm{p}<0.001)$ in extension. Stiffness of the titanium screw/PMMA

17 fixation was significantly greater than the Uniplate 2 construct in this direction. Flexion

18 and lateral bending could not be evaluated in 3 of 6 specimens in the Uniplate 2 group

19 due to failure at the bone/implant interface during extension testing.

20 Conclusions: Fixation with Uniplate 2 was biomechanically inferior to screws/PMMA.

21 Particularly concerning was the incidence of vertebral fracture after several testing

22 cycles. Fixation using the Zero-P and Zero-P VA was unsuccessful due to anatomic

23 constraints in the vertebral column sizes used in this study. 


\section{INTRODUCTION}

25 Canine cervical spondylomyelopathy (CCSM) is a common, naturally occurring and

26 progressive disorder affecting large and giant-breed dogs. The pathophysiology and

27 clinical sequelae of CCSM in dogs are similar to those of compressive cervical

28 myelopathy in humans. ${ }^{1}$

30 Surgical stabilization of the cervical vertebral column in dogs affected with CCSM has

31 become a promising treatment option, and has been largely modeled from human

32 medicine. ${ }^{1-10}$ One option for surgical treatment involves distraction of the affected

33 intervertebral articulation to relieve soft tissue compression of the spinal cord, followed

34 by stabilization to attain bony fusion across the site. While a multitude of techniques and

35 implants have been developed for human cervical fusion, the development of canine

36 spine specific veterinary implants is still in its infancy, with most available literature

37 based on case reports and few biomechanical studies. ${ }^{8-10}$

39 A range of standard veterinary orthopedic implants have been adapted for use in the

40 canine cervical vertebral column. Clinical and biomechanical reports of locking plates

41 used with monocortical screws and monocortical screw/polymethylmethacrylate

42 constructs support their use in dogs. ${ }^{1,5,9-13}$ Avoiding use of PMMA in fixation methods

43 could be beneficial in preventing complications associated with this material including

44 thermal injury during curing, increased risk of infection, cement failure, bulky material

45 with possible soft tissue compression, and difficult removal. ${ }^{1,5,7,13}$ Utilization of locking 
46 plates could eliminate many of the disadvantages of PMMA and offer rigid fixation with

47 a low profile and the ability to use monocortical screws.

49 Two clinical studies have evaluated human fusion plate systems in canine patients. ${ }^{1,10}$

50 Bergman $^{10}$ evaluated a cervical spine locking plate (CSLP; Synthes) combined with a

51 cortical ring allograft and cancellous autograft in 10 dogs. Of the 8 dogs that were

52 available for follow-up, 7 had moderate to complete improvement and did not experience

53 recurrence at long term follow-up (approximately 2.5 years). Trotter ${ }^{1}$ also evaluated the

54 use of CSLP, this time with cancellous block interbody grafting, and reported satisfactory

55 outcomes in 8 of 10 dogs at long term follow-up of 1-5 years. Complications encountered

56 with the use of the CSLP in the canine spine related to the highly variable shape of the

57 ventral aspect of the canine cervical vertebrae which required increased time to contour

58 and bend the plate - it was hypothesized that such changes may contribute to

59 complications such as screw loosening via alteration of the plate's biomechanical

60 efficacy. ${ }^{10}$

61

62 Given the inherent costs associated with manufacturing implants specifically for the

63 canine market, it would clearly be advantageous to be able to make use of standard

64 human implants in dogs. Differences in spinal dimensions and anatomy between humans

65 and dogs make this challenging, especially in the cervical spine. ${ }^{1}$ Locking plate designs

66 offer some advantages in this regard since they do not rely on absolutely accurate

67 contouring between the plate and the underlying bone structures. With this in mind, we 
68 wanted to explore the potential utility of three contemporary locking plate designs: Zero-

69 P and Zero-P VA and Uniplate 2 (DePuy Orthopaedics, Inc., Warsaw, Indiana, USA).

71 The Zero-P implant is a stand-alone implant designed for cervical interbody fusion in

72 humans with degenerative disc disease and spinal stenosis. ${ }^{14}$ It features a radiopaque

73 PEEK interbody spacer of variable height and angle, and a titanium alloy interbody plate

74 that accepts four $2.4 \mathrm{~mm}$ self-tapping titanium alloy locking screws ( 2 screws in cranial

75 and 2 screws in caudal orientation). ${ }^{14}$ (Figures 1 and $2 \mathrm{~A}$ ).

77 The Zero-P VA uses the same PEEK interbody spacer; however, the interbody plate only

78 allows two $3.7 \mathrm{~mm}$ titanium alloy screws for fixation (one screw each for cranial and

79 caudal fixation). ${ }^{15}$ These are variable angle screws that can be oriented in wider

80 trajectories than the locking screws used in the Zero-P, potentially facilitating bony

81 purchase in the vertebral bodies. ${ }^{15}$ (Figures 1 and $2 \mathrm{~B}$ ).

82

83 Several biomechanical reports have been performed in humans to evaluate the Zero-P and

84 Zero-P VA implants for use in cervical interbody fusion. One biomechanical study

85 suggested that a spacer implant with locked screws (Zero P) significantly reduces motion

86 compared to an intact spine, while a variable angle screw spacer failed to provide

87 adequate stabilization for the same type of injury. ${ }^{16}$ Additionally, it was reported that

88 anchored cage implants (the Zero-P) had similar clinical outcomes to that of a cage

89 combined with a plate, but were inferior in stabilization of motion and rates of fusion. ${ }^{17}$

90 
91 The Uniplate 2 system is a low profile 2-hole locking titanium plate developed to hasten

92 operative times and reduce soft tissue dissection around the vertebral body ${ }^{18}$ (Figure 1

93 and 2C). It relies on fixation with only one screw per vertebral level (monovertebral

94 monocortical fixation). Limited reports in the human literature suggest equivalent

95 biomechanical efficacy of the similarly designed Uniplate ${ }^{\mathrm{TM}}$ (Depuy Spine, Raynham,

96 MA, USA) to other plates that utilize one screw per vertebral level. ${ }^{19}$

97

98 To the authors' knowledge, none of these 3 implants have been evaluated for either

99 technical feasibility or for biomechanical effect in the canine cervical spine. If usable and

100 efficacious, they may offer alternative options for dogs requiring cervical distraction and

101 fusion using spine-specific implants. The goal of this study was therefore to evaluate the

102 two Zero-P designs (used with the standard PEEK spacer) and the Uniplate 2 (used with a

103 cortical ring allograft spacer) in the canine cervical vertebral column. Biomechanical

104 testing was performed and the results compared to those of an established monocortical

105 titanium screw(Ti)/PMMA (polymethylmethacrylate)/cortical ring construct. Our

106 hypothesis was that the human implants could be applied to the canine cervical vertebral

107 column and that there would be no significant difference in the stiffness between them

108 and the Titanium/polymethylmethacrylate ring construct. 
109 MATERIALS AND METHODS

110 The study was reviewed and approved by the local Institutional Laboratory Animal Care

111 and Use Committee.

112

113 Tissue Specimens

114 Canine cervical vertebral columns $(\mathrm{C} 2-\mathrm{C} 7)$ were harvested from mature dogs $(\mathrm{n}=18)$

115 that had been euthanatized for reasons unrelated to this study. To be included, dogs had

116 to weigh $20-31 \mathrm{~kg}$. Orthogonal radiographs were obtained to ensure physeal closure and

117 lack of pathology affecting the vertebrae and disk spaces. Vertebral bone mineral density

118 measurements were made by dual - energy X - ray absorptiometry (DEXA) scans (Lunar

119 Prodigy; GE Healthcare, Milwaukee, WI) and these data were used to allocate specimens

120 into three balanced groups. Surrounding soft tissues were resected except for vertebral

121 musculature, joint capsules and ligaments associated with vertebrae C3-C6. Specimens

122 were then wrapped in moist towels, soaked in sterile saline $(0.9 \% \mathrm{NaCl})$ solution, and

123 frozen until testing. Specimens were kept moist using sterile saline solution during

124 processing and testing.

125

126 Biomechanical Testing

127 The cervical vertebral columns were thawed to room temperature, the vertebral motion

128 units (VMUs) between C3-C4 and C5-C6 were immobilized using previously described

129 methods $^{12}$ and an extensometer applied across C4-C5. The operated C4-C5 motion unit

130 was then tested in extension, flexion and right lateral bending using a custom made,

131 four - point bending fixture with a previously reported protocol. ${ }^{12,20}$ Briefly, after a 
132 preload of 5 Newtons $(\mathrm{N})$, testing was conducted under load control at $50 \mathrm{~N} / \mathrm{min}$ to $150 \mathrm{~N}$

133 in flexion and extension and to $100 \mathrm{~N}$ in right lateral bending. Each specimen

134 sequentially underwent 4 full cycles of extension, flexion, and lateral bending with load

135 and displacement data from the $4^{\text {th }}$ cycle data used for analysis when available. Load and

136 extensometer displacement data were used to calculate load-displacement curves for

137 each bending moment of the intact C4-C5 motion unit. Stiffness (N/mm) was determined

138 by calculating the slope of the linear portion of each load-displacement curve. After

139 testing was completed for the intact specimen, the spinal instrumentation was applied

140 (see below) and testing repeated on the instrumented C4-C5 levels.

142 Instrumentation of the C4-C5 motion unit

143 Six specimens were initially used to evaluate the Zero-P and Zero-P VA implants. After a

144 standard ventral approach to the mid cervical spine, a diskectomy was performed at C4-

$145 \mathrm{C} 5$ and the disk space was manually distracted. A 5mm height parallel PEEK ring was

146 inserted into the disk space with the appropriate interbody plate attached.

148 For the Zero-P implant, (DePuy Orthopaedics, Inc., Warsaw, Indiana, USA), a threaded

149 drill guide was used and holes were drilled with a $1.8 \mathrm{~mm}$ drill and the plate secured with

150 four $2.4 \mathrm{~mm}$ stainless steel self-tapping locking screws, 2 placed in the cranial vertebra

151 and 2 in the caudal vertebra. For the Zero-P VA implant, a $2.5 \mathrm{~mm}$ awl with variable

152 angle sleeve was used to create a pilot hole into the caudal endplate bone of C4 and

153 cranial endplate bone of C5 prior to screw insertion. Two $3.7 \mathrm{~mm}$ self-drilling titanium

154 alloy screws were then placed into the cranial and caudal vertebral endplate with a 
155 stardrive screwdriver. None of these 6 spines proved to be stable and further testing of

156 these 2 devices was abandoned (Figures $2 \mathrm{~A}$ and B).

157

158 The Uniplate 2 implant (DePuy Orthopaedics, Inc., Warsaw, Indiana, USA) was

159 successfully deployed in 6 of 6 test specimens. A standard approach to the ventral aspect

160 of C4-C5 was performed. After diskectomy and manual distraction, a cortical ring

161 allograft of appropriate size, harvested previously from canine cadaveric tibiae, was

162 placed into the C4-C5 intervertebral disk space. The Uniplate 2 was placed to span the

163 intervertebral space, ensuring that screw holes and subsequent screws would avoid the

164 disk space. Plate contouring was also used to optimize the fit between the plate and the

165 underlying bone. An awl was used to penetrate the cis cortex and mark position of the

166 screw hole, then a $3.2 \mathrm{~mm}$ drill bit was used to drill through the cis cortex only. A $4.6 \mathrm{~mm}$

167 self-drilling screw was then inserted monocortically into the C4 and C5 vertebral body.

168 The screws were locked via a cam-lock technique using the CAM-LOC and tightened

169 using the CAM Tightener Shaft (DePuy Orthopaedics, Inc) until an audible click

170 occurred, signaling that the proper level of torque had been reached (Figure 2C).

171

172 In order to provide clinical context for these biomechanical tests, comparisons were made

173 against a series of 6 cervical vertebral specimens that were implanted with screw/PMMA

174 and an interbody spacer as part of an earlier study. ${ }^{12}$ These specimens had been harvested

175 under the same inclusion criteria and procedural protocols, and had undergone C4-C5

176 stabilization with cortical ring disk spacer and $\underline{3.5 \mathrm{~mm}}$ monocortical self-tapping titanium 
177

178

179

180 Postoperative Implant Assessment

181 Post-implantation and post-testing orthogonal radiographic projections were used to

182 assess implant position and to identify any evidence of mechanical failure at the implant-

183 bone interface or within the bone itself (Figure 2).

184

185 Statistical Analysis

186 Descriptive statistics were calculated for all variables. Baseline characteristics between

187 the Uniplate 2 and Titanium screw/PMMA fixation were compared using Fisher exact

188 tests for categorical data and Student's t tests for quantitative data. Stiffness was

189 compared between spines fixed with the Uniplate versus titanium screw/PMMA and

190 evaluated among the 3 directional measurements (i.e., extension, flexion, and lateral

191 bending). Ninety-five percent mid-P exact confidence intervals (CI) were calculated for

192 each measurement. Statistical testing was performed using commercially available

193 software (IBM SPSS Statistics, Version 22, International Business Machines Corp.,

194 Armonk, NY) and significance was set at $p<0.05$. 


\section{RESULTS}

\section{Vertebral Specimens}

197 Spines of 6 large breed dogs were used to apply the Zero-P and Zero-P VA to mid and

198 caudal cervical VMUs. The 5mm parallel PEEK cage could be applied without difficulty.

199 Screws into the caudal vertebra appeared well positioned; however, screws in the cranial

200 vertebra did not achieve adequate bony purchase for either implant. Most screws only

201 penetrated a small amount of the caudal endplate of C4 at the ventral aspect. At best, they

202 purchased bone at the caudal base of the transverse process of C4. Fixation was deemed

203 inappropriate and biomechanical testing was not performed.

205 Twelve cervical vertebral column specimens were used for biomechanical testing of the

206 Uniplate $2(n=6)$ and the titanium screw/PMMA construct $(n=6)$. All specimens were

207 from Pit Bull terriers that were skeletally mature, as determined by radiographic evidence

208 of physeal closure, and free of evidence of vertebral pathology. There were 6 intact male

209 and 6 intact female dogs and body weight ranged from $22-30 \mathrm{~kg}$. There was no

210 significant difference in body weight, gender or bone mineral density between these 2

211 groups.

212

213 Biomechanical Testing

214 In the Uniplate 2 group, all of the intact specimens were successfully tested in extension,

215 flexion and lateral bending. The instrumented specimens were all successfully tested in

216 extension, but flexion and lateral bending data were not available in 3 of the fixed 
217 specimens due to overt failure in extension. The titanium screw and PMMA group was

218 successfully evaluated in all directions in both the intact and instrumented specimens.

220 Mean $( \pm \mathrm{SD})$ differences in stiffness were determined for specimens stabilized with the

221 Uniplate and compared to those stabilized with self-tapping titanium cortical screws and 222 PMMA in all directions (Table 1).

223

224 All surgical methods increased stiffness over the unaltered spine $(P<0.001)$. Stiffness of

225 the Ti screw/PMMA fixation method was significantly greater than stiffness achieved

226 with the Uniplate fixation method $(\mathrm{P}<0.001)$ in all measurement directions. Stiffness was

227 also significantly different among measurement direction-extension, flexion and right

228 lateral bending $(\mathrm{P}<0.001)$.

\section{Radiographic Implant Assessment}

231 Cortical ring allografts were seated within the borders of the endplates in all specimens.

232 None of the Uniplate 2 screws penetrated the vertebral canal. Three of the specimens had 233 radiographically apparent failure via fracture of the caudal endplate of $\mathrm{C} 4$. One of these

234 specimens failed catastrophically in the first extension cycle, and further testing was not 235 performed. One specimen exhibited pull-out of the caudal screw at the cranial endplate of

236 C5 with an associated endplate fracture. One specimen appeared to have failure of the

237 screw bone interface as it was grossly unstable during testing despite no radiographic 238 evidence of fracture. 
239 There was no radiographically apparent evidence of failure of the Ti screw/PMMA

240 implants or bone after biomechanical testing. There was evidence of minimal canal 241 penetration $(<2 \mathrm{~mm})$ with 1 of 36 screws. 


\section{DISCUSSION}

243 We compared the mechanical properties of a monocortical screw/PMMA construct to

244 that of the monocortical locking Uniplate 2 construct and found that the Uniplate 2

245 construct was biomechanically inferior to screw/PMMA constructs in cadaveric cervical

246 vertebral column of dogs weighing 22 to $30 \mathrm{~kg}$. Additionally, we found that the Zero-P

247 and Zero-P VA implants could not be applied to the cervical vertebral columns of dogs in

248 this weight group.

250 In human medicine, use of plating for anterior cervical spine fusion is widespread. ${ }^{18}$ Use

251 of bicortical anterior screw fixation in anterior cervical fusion has fallen out of favor due

252 to risks of neurologic compromise which lead to development of constrained cervical

253 plates with locking mechanisms such as the Uniplate 2 system. ${ }^{18}$ The Uniplate 2 design is

254 unique in that it requires just one relatively large screw per vertebral level. This design is

255 meant to address previously reported problems associated with use of two screws per

256 vertebra in diseased or fractured vertebra, and backing out of small screws. ${ }^{21}$ The

257 Uniplate 2's small size also allows for its use in combination with other fixation methods

258 such as cages, while reducing possibilities of dysphagia and/or recurrent nerve paralysis

259 associated with large incisions with aggressive dissection and thermal injury from

260 material such as PMMA. ${ }^{21}$ Other benefits of one screw cervical plates (one

261 screw/vertebral body plate=OSP) compared to the traditional two-screw/vertebral body

262 plate (TSP) include a narrower profile, shorter operation time, and reduction of blood loss

263 and damage to surrounding soft tissues while potentially maintaining the mechanical

264 stability required after fusion procedures. ${ }^{22}$ Possible downfalls of utilizing a single screw 
265 per vertebrae versus 2 screws have been proposed, mainly that a single screw construct

266 may not successfully resist motion as well as a 2 screw construct. ${ }^{22-23}$

268 Several studies in human medicine report biomechanical efficacy of OSP plates of similar

269 design to the Uniplate 2. ${ }^{19,22-24}$ One study evaluated the Uniplate ${ }^{\mathrm{TM}}$ (Depuy Spine,

270 Raynham, MA, USA), an OSP similar to the Uniplate 2, and concluded that there was no

271 significant difference between stiffness in specimens fixed with a OSP versus the

272 traditional TSP. ${ }^{19}$ An evaluation of constructs that utilize 1 screw per vertebral segment

273 versus 2 screws found that, despite the theoretical stability provided by 2 screws, there

274 was no significant biomechanical difference between the 2-screw plate and the 4-screw

275 plates in flexion, extension, lateral bending and axial rotation up to $1.5 \mathrm{Nm} \cdot{ }^{23}$ Another

276 biomechanical study determined that the Uniplate provides satisfactory stabilization

277 (statistically insignificant difference) of cranial cervical spine intervertebral

278 decompression in calf cadavers compared to the more traditional ORION Anterior

279 Cervical Plate System ${ }^{\circledR}$ (Sofamor Danek, Memphis TN). ${ }^{24}$ Finally, in a biomechanical

280 comparison of various OSPs and TSPs, it was found that in lateral bending, the Uniplate

281 had the largest increase in range of motion (ROM) (38\% increase) of all plates tested,

282 while one of the TSPs exhibited the smallest increase in ROM (10\% increase). ${ }^{22}$ This

283 study proposed that the high ROM in lateral bending despite fixation may be due to lack

284 of counter-rotation provided from a second point of fixation (i.e. second screw). While

285 the overall performance of OSPs appears equivalent to that of TSPs in these

286 biomechanical studies, cyclic or fatigue testing to examine long-term outcomes was not

287 performed. 
288 The results from our study in canine cadavers do not support the notion that

289 monovertebral fixation with the Uniplate 2 system is biomechanically equivalent to

290 multiple screw fixation in the canine cervical spine. Our findings are supported by other

291 biomechanical studies in both human and veterinary literature. One study tested

292 triangulated double-screw fixation compared to single-screw instrumentation in anterior

293 spine surgery and found that fixation of the vertebra-device interface is substantially

294 improved by application of the two triangulated screws. ${ }^{25}$ Another study found that

295 stiffness showed a significant linear increase with increasing number of monocortical

296 screws in plate-rod fixation of canine femoral-gap ostectomy models. ${ }^{26}$

298 Furthermore, studies in human medicine demonstrate a high rate of complications in

299 utilization of a OSP system. A case series of humans treated with the Uniplate for

300 anterior cervical fusion demonstrate a high rate of symptomatic pseudarthrosis

301 necessitating revision surgery compared to patients who were treated with a bivertebral

302 screw plating system (4 of 13 cases (31\%) versus 1 of 24 cases (4.4\%), respectively). ${ }^{18}$

303 Development of pseudarthrosis could not be evaluated in our cadaveric study but should

304 be evaluated during prospective studies to determine how complication rates compare.

306 Size of the canine cervical disk spaces is a limiting factor when considering many human

307 cervical interbody spacers for dogs. The small plate design of the Uniplate 2 and the

308 dimensions of PEEK cages of the Zero-P implants appeared to make these implants

309 applicable to the cervical vertebral column of large breed dogs. The Pit bull sized dogs

310 used in this study are on the lower end of the weight spectrum of dogs typically affected 
with CCSM. The weight range of 22-30kg was chosen to allow for direct comparisons between these human cervical implants and previously evaluated implants. ${ }^{12}$

314 The Zero-P and Zero-P VA were initially considered for biomechanical testing and

315 comparison. ${ }^{14,15}$ These implants combine a cervical interbody spacer and screw fixation

316 through an integrated plate. While the $5 \mathrm{~mm}$ parallel PEEK interbody spacers (smallest

317 available) fit well within the confines of large breed canine disk spaces (Pit Bull terrier

318 dogs), the cranially oriented locking screws could not engage the vertebral endplate due

319 to the slant of the canine cervical disk space. As such, while the cages themselves were

320 successfully used in dogs, neither the Zero-P nor Zero-P VA could be implanted stably.

321 Among veterinary implants, the C-Lox device, which has been biomechanically

322 evaluated, works with a similar distraction and fixation concept (disk spacer with

323 incorporated screw fixation). ${ }^{11}$

325 All specimens in the Uniplate 2 group showed failure at the bone-screw interface, with

326 the most common mechanisms of failure being fracture through the caudal aspect of the

327 vertebral body/endplate of the cranial vertebra (Figure 3). A likely explanation for this

328 mode of failure is the hourglass shaped anatomy of the canine cervical vertebral body,

329 which creates a stress riser against the single locking screw. In the Uniplate 2 construct,

330 the locking screws are relatively large compared to the diameter of the vertebral body,

331 and may take up a larger percentage than the traditionally recommended $25 \%$ screw

332 diameter to bone ratio. ${ }^{27-29}$ Larger dogs may have increased vertebral body dimensions,

333 which may decrease the risk of vertebral body fracture.--This, however, would have to be 
334 evaluated by biomechanical studies utilizing larger breed dogs. None of the screws or

335 plates showed macroscopic evidence of failure. As with other locking implants, failure of

336 such constructs often occurs at the screw/bone interface via pull out of the bone or

337 fracture. ${ }^{30-33}$

338

339 One large limitation of this study is that many of the specimens failed prior to the $4^{\text {th }}$

340 cycle in extension, preventing consistency in the cycle used for statistical analysis. The

341 high rate of failure in extension (the first direction tested) also precluded biomechanical

342 evaluation of the Uniplate 2 in flexion and in lateral bending in most specimens. Even

343 when flexion and lateral bending were evaluated, there was concern about compromise of

344 the bone-implant interface and resultant effects on stiffness. We considered changing the

345 order of testing to obtain potentially more valid stiffness values for the other directions.

346 This, however, would have compromised comparison to the testing of the screw/PMMA

347 constructs. Likewise, we considered lowering the load to prevent failure and allow us to

348 test all 3 directions. Other veterinary biomechanical studies have much lower reported

349 load settings but where also done with different testing setups. ${ }^{8,10}$ To be able to directly

350 compare the Uniplate 2 to the titanium screw/PMMA constructs, we decided to maintain

351 the $150 \mathrm{~N}$ load endpoint. Since the necessary stiffness of spinal implants is not known, an

352 argument could be made that $150 \mathrm{~N}$ is too high of a load. However, previous studies have

353 used this load endpoint with other implants using the same testing setup without apparent

354 bone/implant failure. ${ }^{12,20}$ Future studies should focus on larger dogs with increased

355 vertebral dimensions, that are on the higher end of the size spectrum for CCSM, to 
356 determine if these implants have improved performance (Uniplate 2) and improved

357 ability of application (Zero-P, Zero-P VA).

359 Monocortical titanium screw/PMMA constructs using a cortical bone ring as spacer have

360 been biomechanically evaluated in the canine cervical spine and compared to other

361 implant constructs. ${ }^{12}$ This construct allows non-constrained placement of individual

362 screws and ability to adjust the screw insertion location and angle. They are also more

363 cost effective than human cervical fusion plates. While the use of veterinary locking

364 plates has been described for the use of canine cervical stabilization, these plates are not

365 specifically designed for the canine cervical vertebral column. ${ }^{5,8,13}$ Plate length and hole

366 location must be carefully assessed for the individual dog, and the locking mechanism

367 demands a specific screw orientation within the screw hole, which limits the versatility of

368 screw orientation within the plate.

369

370 In conclusion, the results from this study indicate that both the Uniplate 2 and titanium

371 screw/PMMA construct achieve a significant increase in stiffness compared to the

372 unaltered spines. The Uniplate 2, however, is significantly less stiff compared to the

373 screw/PMMA construct and led to bone/screw interface failure in all specimens.

374 Although the data was obtained in an in vitro model, which cannot fully predict in vivo

375 biomechanical behavior, the human clinical implant cannot be recommended for use in

376 Pit bull sized dogs at this time. Future studies should focus on biomechanical tests of both

377 the Uniplate 2 and Zero-P implants in larger dogs, as increased vertebral bone stock may 
378 allow for a decrease in vertebral fracture rate when using the Uniplate 2 and improved

379 application of the Zero-P implants.

380 


\section{DISCLOSURE}

382 The authors report no financial or other conflicts of interest related to this report. 


\section{REFERENCES}

384 1. Trotter EJ: Cervical spine locking plate fixation for treatment of cervical

385 spondylotic myelopathy in large breed dogs. Vet Surg 2009;38:705-718

386

387 2. Queen JP, Coughlan AR, May, C et al: Management of disc-associated wobbler

388 syndrome with a partial slot fenestration and position screw technique.

389 J Small Anim Pract 1998;39:131-136

390

391 3. Jeffrey ND and McKee W: Surgery for disc-associated wobbler syndrome in the dog-

392 an examination of the controversy. J Small Anim Pract 2001;42:574-581

394 4. Sharp N, Wheeler S: Cervical spondylomelopathy, in Wheeler S (ed): Small Animal

395 Spinal Disorders, Diagnosis and Surgery (ed 2). Edinburgh, Elsevier Mosby, 2005, pp $396 \quad 211-246$

398 5. Voss K, Steffen F, Montavon PM: Use of the ComPact unilock system for ventral 399 stabilization procedures of the cervical spine. Vet Comp Orthop Tramatol 2006;19: 21-28 400

401 6. Bruecker K, Seim HB, Withrow S: Clinical evaluation of three surgical methods for 402 treatment of caudal cervical spondylomyelopathy of dogs. Vet Surg 1989;18:197-203 403

404 7. Dixon B, Tomlinson J, Kraus K: Modified distraction-stabilization techniques using 
405 an interbody polymethyl methacrylate plug in dogs with caudal cervical

406 spondylomyelopathy. J Am Vet Med Assoc 1996;208:61-68

407

408 8. Solano MA, Fitzpatrick N, Bertran J: Cervical distraction-stabilization using an

409 intervertebral spacer screw and string-of pearl (SOPTM) plates in 16 dogs with disc-

410 associated wobbler syndrome. Vet Surg 2015;44:627-41

411

412 9. Steffen F, Voss K, Morgan JP: Distraction-fusion for caudal cervical

413 spondylomyelopathy using an intervertebral cage and locking plates in 14 dogs.

$414 \quad$ Vet Surg 2011;40:743-752

415

416 10. Bergman RL, Levine JM, Coates JR et al: Cervical spinal locking plate in

417 combination with cortical ring allograft for a one level fusion in dogs with

418 cervical spondylitic myelopathy. Vet Surg 2008;37:530-536

419

420 11. Schöllhorn B, Bürki A, Stahl C, et al: Comparison of the biomechanical properties

421 of a ventral cervical intervertebral anchored fusion device with locking plate

422 fixation applied to cadaveric canine cervical spines. Vet Surg 2013;42:825-831

423

424 12. Hettlich BF, Allen MJ, Pascetta D, et al: Biomechanical comparison between

425 bicortical pin and monocortical screw/polymethylmethacrylate constructs in the

426 cadaveric canine cervical vertebral column. Vet Surg 2013;42:693-700

427 
428 13. Agnello KA, Kapatkin AS, Garcia TC, et al: Intervertebral biomechanics of locking

429 compression plate monocortical fixation of the canine cervical spine. Vet Surg

$430 \quad 2010 ; 39: 991-1000$

431

432 14. DePuy Synthes Spine: Zero-P and Zero-P chronOS. Zero profile anterior cervical

433 interbody fusion (ACIF) device. Synthes Technique Guide; 1-69

434

435 15. DePuy Synthes Spine: Zero-P VA Variable angle zero-profile anterior cervical

436 interbody fusion (ACIF) device. Synthes Technique Guide 2013; 1-44

438 16. Wojewnik B, Ghanayem AJ, Tsitsopoulos PP, et al: Biomechanical evaluation of a

439 low profile, anchored cervical interbody spacer device in the setting of progressive

440 flexion-distraction injury of the cervical spine. Eur Spine J 2013;22:135-141

441

442 17. Lee Y, Kim, Y and Park, S: Does a zero-profile anchored cage offer additional

443 stabilization as anterior cervical plate? Spine 2015;40:63-70

444

445 18. Dumont TM, Lin CH, Tranmer BI, et al: Pseudarthrosis failures of anterior

446 subaxial cervical spine fusion using a plate with a single screw per vertebral

447 body: a case series. World Neurosurg 2014;82:225-230

448

449 19. Fassett DR, Csaszar DJ, Albert TJ: Anterior cervical plating update. Curr Opin

450 Orthop 2007;18:282-288 
451 20. Hettlich BF, Allen MJ, Glucksman GS, et al. Effect of an intervertebral disk spacer

452 on stiffness after monocortical screw/polymethylmethacrylate fixation in stimulated and

453 cadaveric canine cervical vertebral columns. Vet Surg 2014;1-7

454

455 21. Lin PS: United States Patent Application Publication: Uniplate Cervical device

$456 \quad 2004 ; 1-6$

457

458 22. Hunter W, Bucklen B, Muzumdar A, et al: A comparative biomechanical study of

459 traditional and in-line plating systems following immediate stabilization of single and bi-

460 level cervical segments. Clin Biomech 2012;27:84-90

461

462 23. Crawford N, Scholz M, Reyes PM, et al. Biomechanics of one-level anterior cervical

463 discectomy and plating using two screws versus four screws. Spine J 2011;11:234-240

464

465 24. Zhang F, Lu J, Zhao J, et al. Uniplate with the Orion anterior cervical plate fixation

466 biomechanics single gap study. Chin J Orthop Surg 2008;16:1490-2

467

468 25. Ogon M, Krismer C, Sterzinger M, et al: Comparison between single-screw and

469 triangulated, double-screw fixation in anterior spine surgery: A biomechanical

470 test. J OrthopTrauma 2003;18:488-493

471 
472 26. Delisser PJ, McCombe GP, Trask RS, et al: Ex vivo evaluation of the biomechanical

473 effect of varying monocortical screw numbers on a plate-rod canine femoral gap model.

474 Vet Comp Orthop Traumatol 2013;3:177-185

475

476 27. Poitout DG: Biomaterials used in orthopedics. in Poitout DG (ed): Biomechanics and

477 biomaterials in orthopedics. London, UK, Springer, 2004,15-21

478

479 28. Johnson AL, Houlton JEF, Vannini R: Implants and materials in fracture fixation. in 480 AO principles of fracture management in the dog and cat. Davos Platz, Switzerland, AO

481 Publishing, Stuttgart, Germany, Thieme, 2005;477-487

482

483 29. Toombs JP: Fractures of the radial diaphysis. in AO principles of fracture

484 management in the dog and cat. Davos Platz, Switzerland, AO Publishing, Stuttgart, 485 Germany, Thieme, 2005;236-251

486

487 30. Malenfant RC and Sod GA: In vitro biomechanical comparison of 3.5 string of pearl

488 plate fixation to 3.5 locking compression plate fixation in a canine fracture gap model.

489 Vet Surg 2014;43:465-470

490

491 31. Schneider K, Oh J, Zderic, I et al: What is the underlying mechanism for the failure

492 mode observed in the proximal femoral locking compression plate? A biomechanical

493 study. Injury 2015;46:1483-90

494 
495 32. Forward DP, Doro CJ, O’Toole, RV et al: A biomechanical comparison of a locking

496 plate, a nail, and a $95^{\circ}$ angled blade plate for fixation of subtrochanteric femoral fractures.

497 J Orthop Trauma 2012;26:334-340

498

499 33. Thapa N, Prayson M and Goswami T: A failure study of a locking compression plate

500 implant. Case Stud Eng Fail Anal 3 2015:68-72 
501 FIGURE LEGENDS

502 Figure 1. Photographs of each implant in ventral and axial views (middle and right) and 503 showcasing individual components (left).

504

505 Figure 2. Lateral and ventrodorsal radiographic projections of canine C4-C5 vertebrae

506 instrumented with 3 human cervical fusion implants. A: Zero-P with PEEK interbody

507 cage, titanium interbody plate and four $2.4 \mathrm{~mm}$ stainless steel locking screws. B: Zero-P

508 VA with PEEK interbody cage, titanium interbody plate and two self-drilling $3.7 \mathrm{~mm}$

509 variable angle titanium screws. C: Uniplate 2 with cortical ring disk spacer and 2 self-

510 drilling $4.6 \mathrm{~mm}$ titanium screws locked using the CAM-LOC.

511

$512 \quad$ Figure 3.

513 Lateral radiographic projections of C4-C5 vertebrae demonstrating 2 types of implant

514 failures after testing of the Uniplate 2/cortical ring constructs. A: pullout of the caudal

515 screw with a fracture in the cranial endplate of C5. B: fracture of the caudal endplate of

516 C4. 
517 Table 1 Absolute mean $( \pm \mathrm{SD})$ differences in stiffness from pre- to post-fixation for 518 Uniplate 2 versus Ti screw/PMMA [\% of specimens successfully tested]

\begin{tabular}{||l|l|l|l|}
\hline Fixation method & $\begin{array}{l}\text { Extension } \\
(\mathrm{N} / \mathrm{mm})\end{array}$ & Flexion $(\mathrm{N} / \mathrm{mm})$ & $\begin{array}{l}\text { Right Lateral } \\
\text { Bending }(\mathrm{N} / \mathrm{mm})\end{array}$ \\
\hline Uniplate 2 & $115.08(47.09)$ & $61.72(17.93)$ & $18.79(10.31)$ \\
& {$[100 \%]$} & {$[50 \%]$} & {$[50 \%]$} \\
\hline Ti screw/PMMA & $\begin{array}{l}137.06(4.10) \\
{[100 \%]}\end{array}$ & $\begin{array}{l}313.6(83.52) \\
{[100 \%]}\end{array}$ & $\begin{array}{l}327.76(64.21) \\
{[100 \%]}\end{array}$ \\
\hline
\end{tabular}

519 


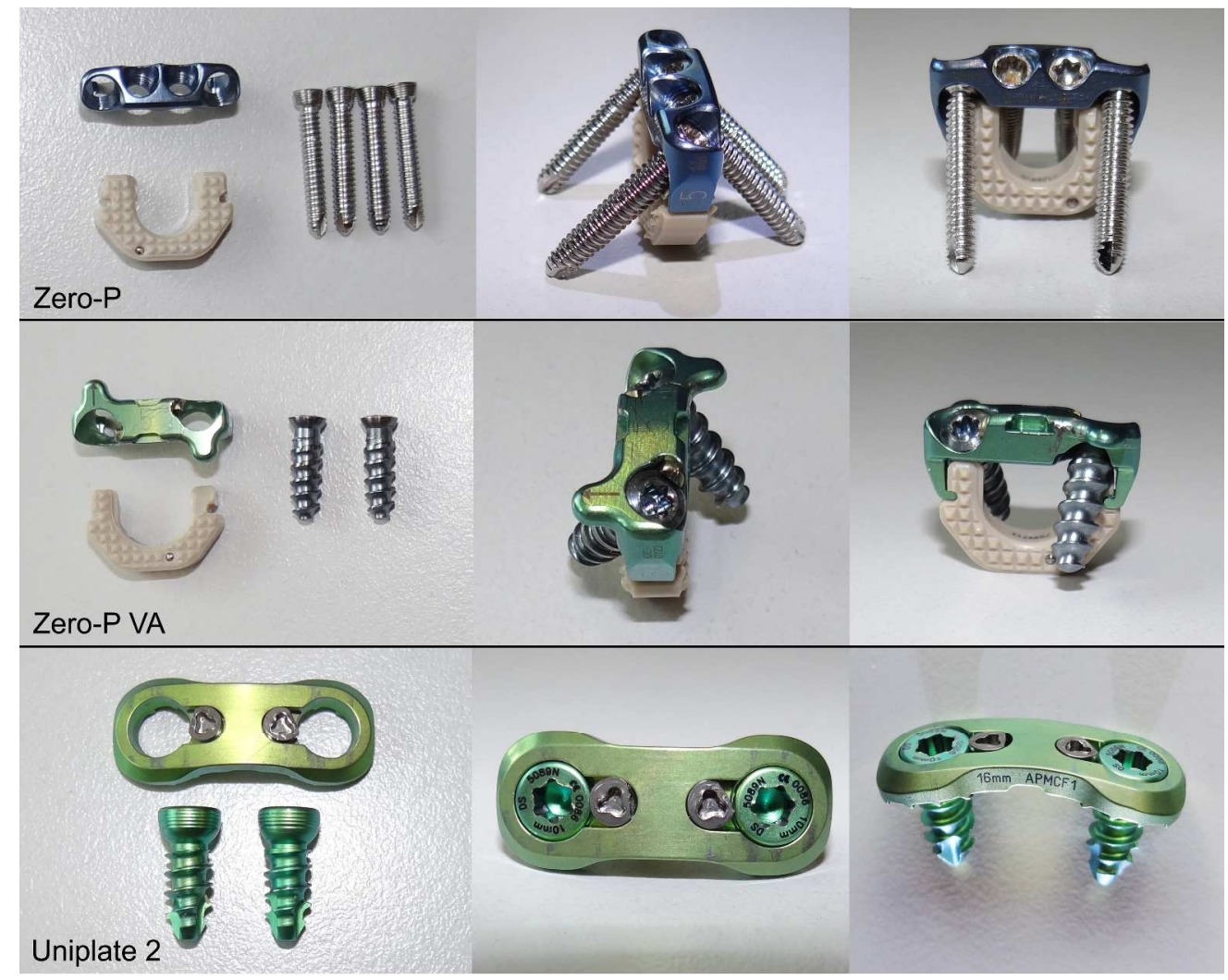

Photographs of each implant in ventral and axial views (middle and right) and showcasing individual components (left). $1321 \times 1060 \mathrm{~mm}(96 \times 96 \mathrm{DPI})$ 

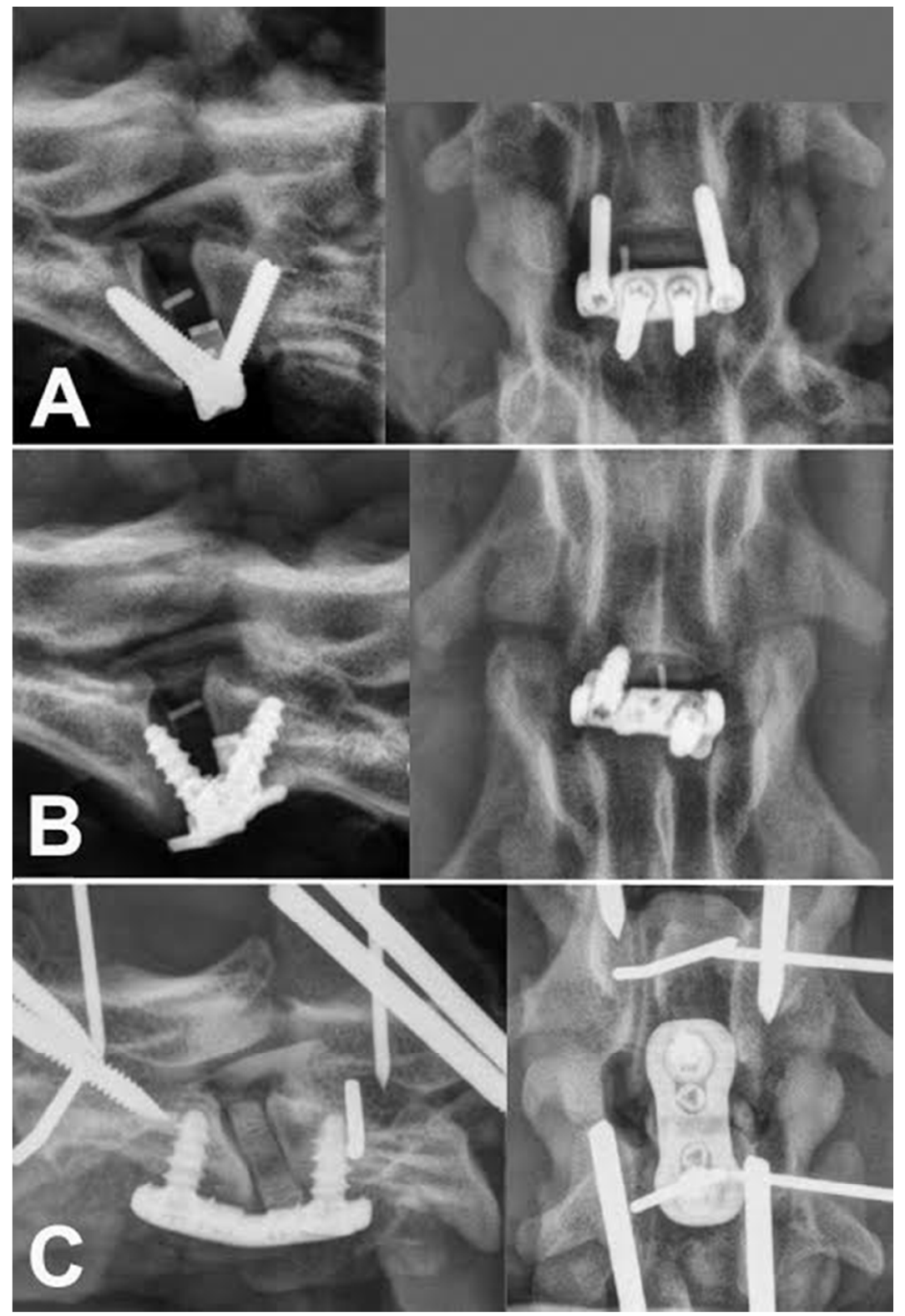

Lateral and ventrodorsal radiographic projections of canine C4-C5 vertebrae instrumented with 3 human cervical fusion implants. A: Zero-P with PEEK interbody cage, titanium interbody plate and four $2.4 \mathrm{~mm}$ stainless steel locking screws. B: Zero-P VA with PEEK interbody cage, titanium interbody plate and two selfdrilling $3.7 \mathrm{~mm}$ variable angle titanium screws. C: Uniplate 2 with cortical ring disk spacer and 2 self-drilling 4.6mm titanium screws locked using the CAM-LOC. $168 \times 249 \mathrm{~mm}(72 \times 72 \mathrm{DPI})$ 


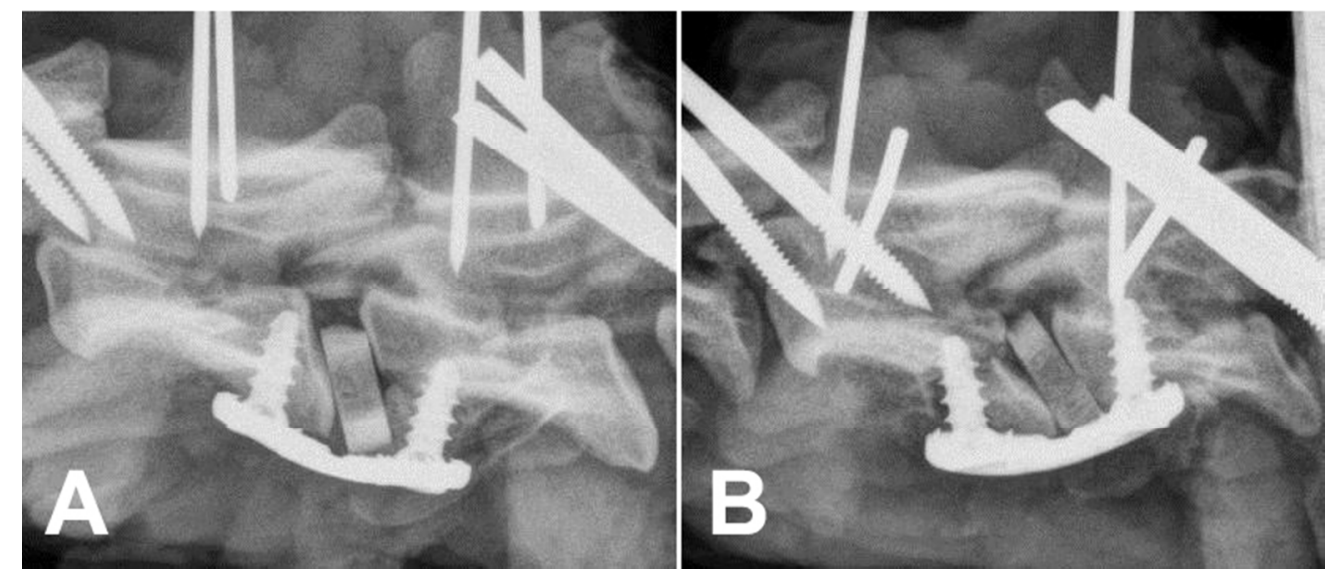

Lateral radiographic projections of C4-C5 vertebrae demonstrating 2 types of implant failures after testing of the Uniplate 2/cortical ring constructs. A: pullout of the caudal screw with a fracture in the cranial endplate of C5. B: fracture of the caudal endplate of $\mathrm{C} 4$.

$218 \times 93 \mathrm{~mm}(96 \times 96 \mathrm{DPI})$ 